\title{
Jump Rates for Point Defects in Special Positions Held by a Trapping Center of Noncubic Symmetry
}

\author{
H. S. Peiser and J. B. Wachtman, Jr. \\ Institute for Materials Research, National Bureau of Standards, Washington, D.C. 20234
}

(February 14, 1968)

\begin{abstract}
In a previous paper [J. Phys. Chem. Solids 27, 975 (1966)] the authors have discussed symmetry conditions for equivalence of jump rates operative in point-defect motion between crystallographically equivalent general sites neighboring a trapping center. The treatment is here extended to equivalent special sites to show that the total number of inequivalent jump rates can be expressed as $\left(N_{t} / N_{d}\right)-q_{e}-1$ where $N_{t}$ and $N_{d}$ are the orders of the symmetry groups of the trap and defect respectively and where $q_{e}$ is the number of independently effective pairs of nonself-inverse symmetry operators (paired with their inverses) all in the symmetry group of the trap. The number $q_{e}$ can be obtained by counting any nonself-inverse operator pair, an element of which, when multiplied by any element of the defect symmetry, equals neither its own inverse nor an element of a pair previously counted.

For thermally activated relaxation processes expressions apply for maximum and minimum numbers of jump frequencies involved in complete and partial relaxation processes. For complete relaxation the maximum number is the number of prime factors in the ratio of the order of the symmetry group of the trap to that of the defect group. The minimum number is the minimum number of generators that will raise the defect position symmetry to that of the trap.
\end{abstract}

Key Words: Crystal symmetry, equivalent sites, jump rates, point defects, relaxation, trapping center.

\section{Introduction}

In a recent publication [1] ${ }^{1}$ we have discussed symmetry conditions for equivalence of jump rates operative in point-defect motion between crystallographically equivalent sites with a view toward an assessment of the extent to which such motion can account for relaxation and diffusion phenomena observable in crystals. Closely related problems have been treated by A. S. Nowick [2] and in papers referred to by him. Our treatment does not depend on linearity of response to an outside influence and is believed to have rather general applicability. In the quoted paper [1] it was applied to a highly specialized situation governed by four restrictions; the partial removal of the second restriction being the contribution in this paper.

Restriction 1: The point defect, though itself relatively mobile, was held to an immobile trapping center, the defect remaining free only to jump between symmetry-equivalent sites on a shell neighboring the trapping center. The effect of this restriction was to reduce a problem concerned with an infinite number of possible defect sites to one concerned with the finite point groups of the trap, $G_{t}$ and $G_{t}^{\prime}$ in the initial and

${ }^{1}$ Figures in brackets indicate literature references at the end of this paper. strained conditions, respectively (all basic notation is summarized in table 1). The corresponding point-group orders are designated $N_{t}$ and $N_{t}^{\prime}$. Both groups are subgroups of the corresponding crystal space groups, and of the crystal point groups $G$ and $G^{\prime}$ of $\operatorname{order} N$ and $N^{\prime}$, respectively.

Restriction 2: The point group of the point-defect site in the unstrained crystal, $G_{d}$, was restricted to the identity 1. In the strained crystal the point group of the defect, $G_{d}^{\prime}$, was necessarily 1 , too, because by simple symmetry postulate [1] $G_{d}^{\prime} \subseteq G_{d}$. We here relax this restriction to allow the site symmetry of the defect to differ from the identity and we refer to its symmetry as symmetry of specialization. $G_{d}$, of order $N_{d}$, must be a noncentrosymmetric subgroup of $G_{t}$ because the trapping center neighbors the defect on only one side. By the correspondence of the shell of sites around the trap with the construction sphere for a stereographic projection, we deduce that the only permitted $G_{d}$ are the ten symmetries achievable on the surface of a sphere (see tables 3 and 4 in reference [3]), which in turn are isomorphous with the ten two-dimensional diperiodic point groups [4]. Although in three dimensions the presence of the trap imposed the limitation of lack of a symmetry center, this limitation does not apply to the isomorphous two-dimensional groups. 
TABLE 1. Symbols used for point groups, their orders as well as for individual operators, defect sites and paths

\begin{tabular}{l|l|l|l|l}
\hline \multicolumn{2}{c|}{ Crystal } & \multicolumn{1}{c|}{$\begin{array}{c}\text { Point } \\
\text { group }\end{array}$} & Order & $\begin{array}{c}\text { Symmetry } \\
\text { operator } \\
\text { in group }\end{array}$ \\
\hline \multirow{5}{*}{ Crystal } & Unstrained & $G$ & $N$ & $S$ \\
& Strained & $G^{\prime}$ & $N^{\prime}$ & $S^{\prime}$ \\
Trap & Unstrained & $G_{t}$ & $N_{t}$ & $S_{t}$ \\
& Strained & $G_{t}^{\prime}$ & $N_{t}^{\prime}$ & $S_{t}^{\prime}$ \\
& Unstrained & $G_{d}$ & $N_{d}$ & $S_{d}$ \\
& Strained & $G_{d}^{\prime}$ & $N_{d}^{\prime}$ & $S_{d}^{\prime}$ \\
\hline
\end{tabular}

$G_{d}^{\prime} \subseteq G_{d} \subseteq G_{t} \subseteq G \supseteq G^{\prime} \supseteq G_{t}^{\prime} \supseteq G_{d}^{\prime}$ and $G_{t} \supseteq G_{t}^{\prime}$

where $\supseteq$ indicates a subgroup relationship of the right group to the one written to the left of that symbol.

An $S$ could be a $1,2,3,4,6, \overline{1}, \overline{2}, \overline{3}, \overline{4}$ or $\overline{6}$ axial operator to the first positive or negative power.

$S^{-1}$ is specifically an inverse of an $S$.

$\bar{S}$ is specifically an inversion axis operator.

$\tilde{S}$ is specifically a rotation axis operator.

$|S|$ is the order of the axis.

Additional suffixes $1,2,3 \ldots$ are used to distinguish different operators.

[uvw] is a direction $\epsilon\langle u v w\rangle$ where $\epsilon$ means "an element of."

$S[u v w]$ is the direction $\epsilon\langle u v w\rangle$ to which $[u v w]$ is transformed by operator $S$

$S_{2} S_{1}[u v w]=S_{3}[u v w]$ when the successive operations $S_{1}$ followed by $S_{2}$ is equivalent to the single operation of $S_{3}$.

$[u v w] \rightarrow S[u v w]$ is a defined path from a site defined by $[u v w]$ to one defined by $S[u v w]$.

$[u v w]^{\wedge} S$ is the angle between $[u v w]$ and the direction of $S$.

Indeed the two-dimensional environment of a defect on the surface of the shell and on its tangent plane may be two-dimensionally centrosymmetric.

There exist alternative ways of expressing all possible symmetry operators $S$. Most systems commonly used allow, in addition to 1 , any of the crystallographically permitted rotation axes $2,3,4$, or 6 . To complete a self-consistent set of distinct possible operators $S$ in $G$ we will use inversion axes $\overline{1}, \overline{2}, \overline{3}, \overline{4}$, and $\overline{6}$. Where the order of the operator, $|S|$, defined by the smallest positive integer for which $S^{|S|}=1$, is greater than 2 (that is for $3,4,6, \overline{3}, \overline{4}$, and $\overline{6}$ ) the operator is nonself-inverse and its inverse is distinct. Nevertheless, if $S$ is an element of $G, S^{-1}$ is, too, i.e., $S \epsilon G \leftrightarrow S^{-1} \epsilon G$. Of the inversion axes only $\overline{2}$, equivalent to a mirror plane, $m$, provides a possible $S_{d} \epsilon G_{d} . \overline{2}$ differs from all rotation-axial $S_{d}$, denoted $\widetilde{S}_{d}$, in that $\overline{2}_{d}$ provides a locus of an infinite number of coplanar directions corresponding to defect sites all of which have $G_{d} \supseteq \overline{2}$, whereas a crystal can only possess a small number of sites with rotational symmetry of specialization. (It is not implied that a defect with ${ }_{d}$ has a degree of freedom of motion along the mirror plane perpendicular to $\overline{2}_{d}$. The sites on $\overline{2}_{d}$ are not in general symmetry related and so can have differing free-energy characteristics.) The possible $G_{d}$ are then the five $\tilde{S}_{d}$ alone plus the same five with a perpendicular $\overline{2}_{d}$ axis $\left(\tilde{S}_{d} / \overline{2}_{d}\right)$. Thus only ten defect groups can occur: $1,2,3,4,6, m, 2 m, 3 m, 4 m$, and $6 m$. The effect of the second restriction imposed in our previous paper [1] was further to reduce consideration to equivalent general sites of the point defect. With $G_{d}=1$, necessarily, the number of equivalent defect sites was always $N_{t}$.

Each site can be defined by a $[u v w] \epsilon\langle u v w\rangle$ where the $u, v, w$ numbers need not be simple integers. Equality of two directions represented by $\left[u_{1}, v_{1}, w_{1}\right]$ and $\left[u_{2}, v_{2}, w_{2}\right]$ is assured by the conditions that the magnitude and sign of any two of the three ratios $\frac{u}{v}, \frac{v}{w}, \frac{w}{u}$ be the same in the two cases and that the sign of one of the three symbols $u, v, w$ be the same. Every element of $\langle u v w\rangle$ can be described as $S_{t}[u v w]$, the direction generated by $S_{t} \epsilon G_{t}$ acting on [uvw]. A path indicated by $[u v w] \rightarrow S_{t}[u v w]$ will refer to a path from a defect site $[u v w]$ to another site $S_{t}[u v w]$. Of the infinite number of such geometric paths a choice can be made to fulfill some specific conditions. If the notation were applied to crystal-chemical networks, it might be adequate to designate the straight-line path. In other applications a great-circle path is satisfactory. In our work here, we mean the path which has the largest jump rate, which, in turn, approximates that of minimum free-energy barrier. The path for largest jump rate need not be straight, nor lie on the surface of the shell containing the sites and has no single geometric representation to justify fully the use of the singular word "path" which, nevertheless, we use. One may thirk of a path with a finite not-sharply delineated width. The removal of restriction 2 will necessitate a further reexamination of the concept of the path (see sec. 2).

In our previous work [1], for which always $G_{d}=1$ and for which the number of sites, consequently, equaled the number of elements in $\langle u v w\rangle$, i.e., $N_{t}$, the number of nonzero paths emanating from [uvw] was always $N_{t}-1$. Symmetry requires that these paths from $[u v w]$ are fully representative of all paths relevant to $\langle u v w\rangle$. This rule will apply equally for $N_{d}>1$.

The aim of the present paper is then to remove this second restriction, but we simplify our task by restricting discussion to noncubic trap sites $G_{t}$.

Restriction 3: We have assumed that (in our new nomenclature)

$$
[u v w] \rightarrow S_{t}[u v w]=[u v w] \rightarrow S_{t}^{-1}[u v w]
$$

even when $\left|S_{t}\right|>2$ that is when

$$
[u v w] \rightarrow S_{t}[u v w] \neq \equiv[u v w] \rightarrow S_{t}^{-1}[u v w] .
$$

This eq (1) will hold for most physical models one can invent. In our previous paper [1] we have in fact shown that the equation must hold for all steady-state conditions under which the principle of detailed population balance is applicable. Our treatment, though in some ways complicated by this restriction, would lose likely contact with possible experiments if we 
removed it. We, therefore, retain the condition imposed by eq (1) throughout this paper.

In our previous paper [1] we found that for each pair of nonself-inverse operators $S$ and $S^{-1}$, the number $J$ of nonequivalent paths from [uvw] was reduced by one. If $q$ is the number of such $S$ pairs $(|S|>2)$

$$
J=N_{t}-q-1
$$

Even after completely upsetting the population in equivalent sites by an externally imposed influence, relaxation by a thermally activated process does not, in general, require all the nonequivalent jump rates to be activated. It is in general impossible to state a number of needed jump rates even for a given $G_{t}$. It was, however, possible [1] to derive simple expressions for the minimum number $J_{\min }$, and the maximum number $J_{\max }$ needed completely to relax a system. The minimum number, $J_{\min }$, of nonequivalent jump rates needed completely to relax a system in which the population of defects over equivalent sites had been completely upset was the rank of $G_{t}$ (the minimum number of operators whose products and powers constitute that group). In most point groups the experimentally observable number of jump rates could be larger than $J_{\min }$ depending on the relative magnitude of the jump rates. There was, however, a usefully restrictive maximum number $J_{\max }$ of nonequivalent jump rates equal to the number of prime factors in $N_{t}$. Similarly, simple rules could be formulated for partially upset populations. If $N_{t}^{\prime}$ of the $N_{t}$ initially equivalent sites remained equivalent through a postulated sequence of influences, the minimum number of jump rates, $J_{\min }$, needed for relaxation, was the minimum number of nonequivalent symmetry operators that would raise $G_{t}^{\prime}$ of order $N_{t}^{\prime}$ to the initial point group $G_{t}$.

The maximum number was the number of prime factors in $\left(N_{t} / N_{t}^{\prime}\right)$. We presented [1] tabulations of maximum and minimum numbers of jump rates. Experiments designed to observe these points must be carried out at temperatures high enough so that an adequate number of jump rates are above the level of observation, so that point defects can attain equilibrium distributions in finite time.

Restriction 4: Along paths emanating from a given $[u v w]$ predictable cross-over positions may occur with equivalent paths emanating from other elements of $\langle u v w\rangle$. Some crossings must be exact, some may only be approximate, because of limited variability of the radial distance from the trap. As a result some paths must have equal, approximately equal, or lower freeenergy barriers than others also emanating from [uvw]. These inequalities have not been discussed in detail, nor are they further discussed in this paper. It should be realized, however, that the effect of these inequalities will be to lower the number of experimentally observable distinct jump rates. Accidental equalities of jump rates under specific conditions and within any given experimental precision need not be discussed in the present context.

\section{Consequences of Symmetry of Specializa- tion for the Defect Sites}

By analogy with the definition of general position in crystallography as the set of symmetry-related positions each of which is left invariant by no other symmetry operator $S$ than the identity, 1, we will use "general direction" to define the $\langle u v w\rangle$ set if a $[u v w]$ $\epsilon\langle u v w\rangle$ is not invariant under any operator $S_{t}$ other than the identity. Similarly "special direction" will signify the $\langle u v w\rangle$ set if at least one $S_{t}$ (other than the identity, 1) will leave a given [uvw] invariant. The special direction characterizes the symmetry properties of a special position set of a point defect confined to a trap. The stereographic representation of a special direction set $\langle u v w\rangle$ can easily be visualized with the help of the steoregraphic representation of the general direction set for the given $G_{t}$ [3]. The positions of the general set $\langle u v w\rangle$ are moved, in conformity with the symmetry requirements, on the stereogram into coalescence on the stereographic representation of the defect symmetry group $G_{d}$. $\left(\frac{N_{t}}{N_{d}}-1\right)$ paths will then emanate from any $[u v w]$. The direction $S_{d}[u v w] \equiv[u v w]$ so that generally for $S_{t} \notin S_{d}$ :

$$
S_{t}[u v w] \equiv S_{t} S_{d}[u v w]
$$

where the product $S_{t} S_{d}$ signifies the operation required by $S_{d}$ followed by that of $S_{t}$, a product which must equal an operator $S_{t 1} \epsilon \mathrm{G}_{t}$; where $S_{t 1} \neq S_{t}$ unless $S_{d}=1$. We, therefore, note that for special positions any one direction of the set $\langle u v w\rangle$ can be written in terms of a given $[u v w]$ in $N_{d}$ ways. All will be of the type $S_{t}[u v w]$. A given path $[u v w] \rightarrow S_{t}[u v w]$ can also be written in $N_{d}$ ways referring to identically the same path by changing only the operator $S_{t}$. This is illustrated for the case $\mathrm{G}_{t}=\overline{6} m 2, \mathrm{G}_{d}=\overline{2}_{\perp} \quad\left(\overline{2}_{\perp}\right.$ signifies $\overline{2} \perp \overline{6}$ ) in figure 1 with its accompanying table.

The concept of the path itself needs to be examined once more also by reference to figure 1 . Reference is made to the path $[u v w] \rightarrow \overline{2}_{\|}[u v w]$ for which $S_{d}=\overline{2}$ leaves both $[u v w]$ and $\overline{2}_{\|}[u v w]$ invariant. For all models for which the geometric path is permitted to depart from the mirror plane of specialization, there will be symmetrically equal geometric realizations of that path on the two sides of the symmetry plane, $\overline{2}_{d}$. The $2_{[1010]}$ axis relating $[u v w]$ to $\overline{2}_{\|}[u v w]$ has a similar effect on any path not going through the angle bisector. Although we are aware of this plurality of some geometric paths $[u v w] \rightarrow S_{t}[u v w]$ we feel justified in continuing to use the singular word path giving rise to one jump rate. For a given free-energy barrier a geometric path plurality will slightly increase the jump rate, but that enhanced jump rate will be a characteristic of the system, namely, that appropriate for the path $[u v w] \rightarrow S_{t}[u v w]$. The geometric plurality of paths does not introduce complexities any more than did the path width discussed in the introduction. In fact a wider path with energetically 


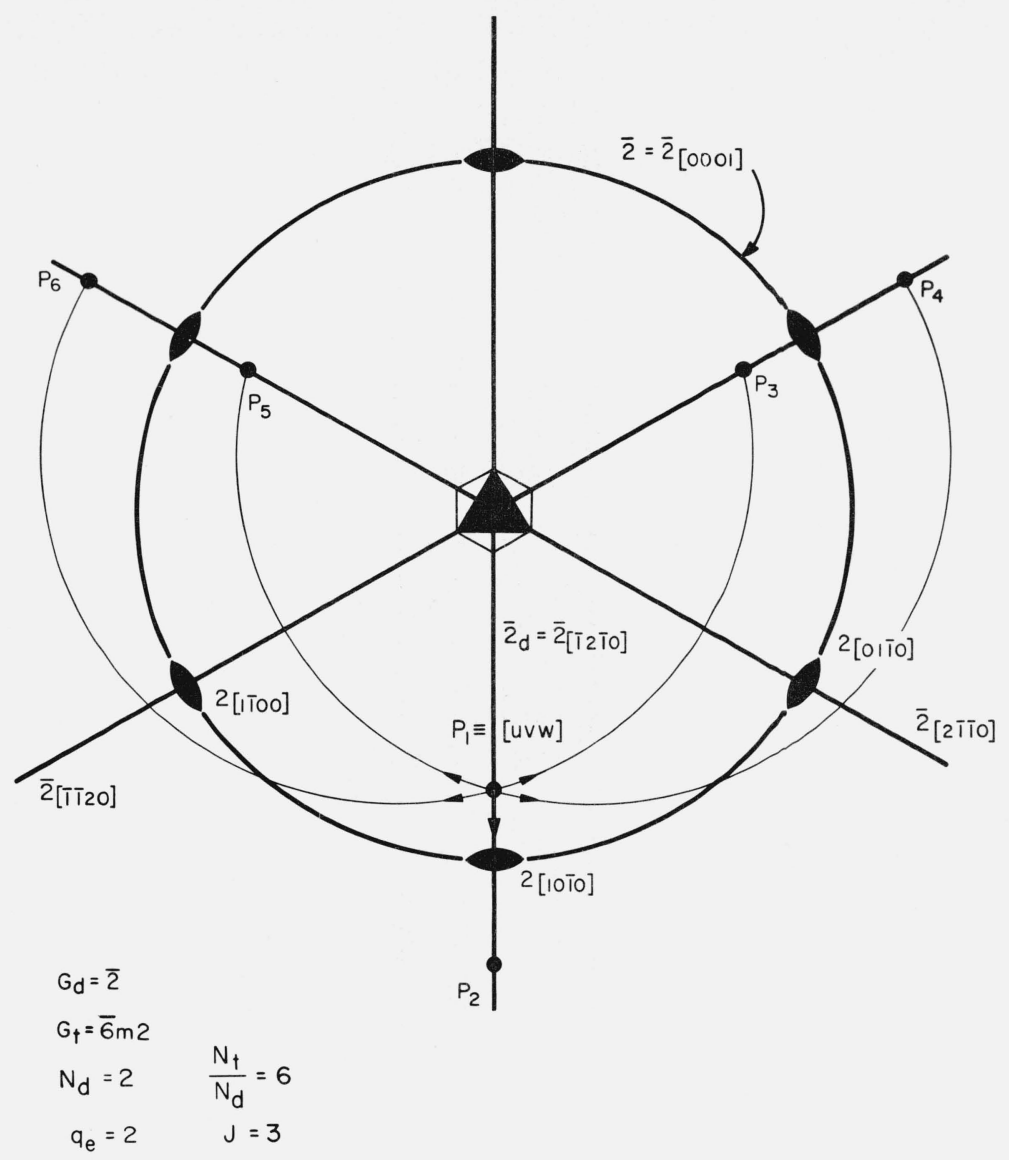

\begin{tabular}{|c|c|c|c|c|c|}
\hline Point & $\begin{array}{l}\text { Two } \\
\text { Methods of } \\
\text { Generation } \\
\text { from } \mathrm{P}_{1}\end{array}$ & Path & $\begin{array}{l}\text { Equivalent } \\
\text { to Path }\end{array}$ & $\begin{array}{l}\text { Equiv } \\
\text { Self-inverse } \\
\text { Operation }\end{array}$ & $\begin{array}{l}\text { lence by } \\
\text { Non } \\
\text { Self-inverse } \\
\text { Pair }\end{array}$ \\
\hline$P_{1}$ & $\begin{array}{c}1 \text { [uvw] } \\
\overline{2}[\overline{1} 2 \overline{1} 0][\mathrm{uvw}]\end{array}$ & - & - & - & - \\
\hline $\mathrm{P}_{2}$ & $\begin{array}{l}\overline{2}_{[0001]^{[\mathrm{uvw}]}} \\
{ }^{2}[10 \overline{1} 0]^{[\mathrm{uvw}]}\end{array}$ & $\mathrm{P}_{1} \rightarrow \mathrm{P}_{2}$ & None & - & 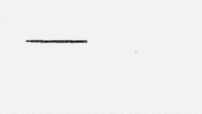 \\
\hline $\mathrm{P}_{3}$ & $\begin{array}{c}\overline{2}_{[2 \overline{1} \overline{1} 0]^{[\mathrm{uvw}]}} \\
3[\mathrm{uvw}] \\
\end{array}$ & $\mathrm{P}_{1} \rightarrow \mathrm{P}_{3}$ & $\mathrm{P}_{1} \rightarrow \mathrm{P}_{5}$ & $\overline{2}_{\mathrm{d}}$ & $3,3^{-1}$ \\
\hline $\mathrm{P}_{4}$ & $\begin{array}{c}{ }^{2}{ }_{01 \overline{1} 0]^{\text {[uvw] }}} \\
6^{-1} \text { [uvw] }^{-1}\end{array}$ & $P_{1} \rightarrow P_{4}$ & $P_{1} \rightarrow P_{6}$ & $\overline{2}_{\mathrm{d}}$ & $\overline{6}, \overline{6}^{-1}$ \\
\hline $\mathrm{P}_{5}$ & $\begin{array}{c}\overline{2}_{[\overline{1} \overline{1} 20]^{[\mathrm{uvw}]}} 3^{-1} \text { [uvw] }^{2} \\
\end{array}$ & $P_{1} \rightarrow P_{5}$ & $\mathrm{P}_{1} \rightarrow \mathrm{P}_{3}$ & $\overline{2}_{\mathrm{d}}$ & $3,3^{-1}$ \\
\hline $\mathrm{P}_{6}$ & $\begin{array}{l}{ }^{2}[1 \overline{1} 00]^{[\mathrm{uvw}]} \\
\overline{6}[\mathrm{uvw}]\end{array}$ & $P_{1} \rightarrow P_{6}$ & $P_{1} \rightarrow P_{4}$ & $\overline{2}_{\mathrm{d}}$ & $\overline{6}, \overline{6}^{-1}$ \\
\hline
\end{tabular}

FIGURE 1. Equivalence of paths from a special position on a mirror plane in point group $\overline{6} \mathrm{~m} 2$, for a defect on a mirror plane, $\mathrm{m}=\overline{2}$. 
shallower sides will in the same way lead to lower jump rates.

In the next section we shall obtain the number of independent paths by a procedure involving the counting of the effective number of nonself-inverse operator pairs. We conclude the present section by describing an alternate procedure involving the counting of certain distinct operator product sets; this alternate procedure is perhaps not as easy to apply to specific cases but provides a different point of view. We begin by noting that we need only consider the paths emanating from any one $[u v w]$ and that all paths emanating from a given $[u v w]$ are of the form $[u v w] \rightarrow S_{t}[u v w]$ where $S_{t} \neq G_{d}$ because [uvw] is invariant under all operators in $G_{d}$. For two paths to be equal as expressed by $[u v w] \rightarrow S_{t 1}[u v w]=[u v w] \rightarrow S_{t 2}[u v w]$ the operators must satisfy either of two conditions. First, equality of paths is assured if $S_{t 2}=S_{t 1}^{-1}$ and $S_{t 1}$ is nonself-inverse; this is the only case which can occur for general $[u v w]$ and was previously treated for general sites. Second, if $S_{d} \epsilon G_{d}$ and

$$
S_{t 2}=S_{d} S_{t 1} \text { or } S_{t 2}=S_{d} S_{t 1}^{-1}
$$

equality of paths is assured (note that the first condition is contained in the second by taking $S_{d}$ to be the identity). This second condition holds because an operator $S_{d}$ which satisfies this condition will leave $[u v w]$ unmoved and will take $S_{t 1}[u v w]$ into $S_{t 2}[u v w]$. By considering each of the possible operations $S_{d}$ in turn one can see that each point of the path $[u v w] \rightarrow S_{t 1}[u v w]$ is carried into the corresponding path $[u v w] \rightarrow S_{t 2}[u v w]$ so that the paths are equivalent and must have equal jump rates. This argument justifies the following procedure for determining the number of independent paths, $J$ : list all of the $S_{t} \notin \mathrm{G}_{d}$. For each $S_{t}$ form the set of operator products $S_{d} S_{t}$ and $S_{d} S_{t}^{-1}$ using all $S_{d} \epsilon G_{d} . J$ is equal to the number of distinct sets.

\section{The Number of Distinct Jump Rates in Terms of the Effective Number of Non- self-Inverse Operators}

In this section we shall first show that only nonself-inverse operators need be considered in determining the number of equivalent paths because even though self-inverse operators do in certain instances relate pairs of paths emanating from special positions their effect is always duplicated by the action of nonself-inverse operators. We then consider the fact that distinct pairs of nonself-inverse operators sometimes coincide in their effect on paths emanating from special positions or fail to relate distinct paths so that the effective number of nonselfinverse operator pairs is sometimes less than the total number of distinct pairs.

Two physically distinct paths emanating from [uvw] may be equalized by self-inverse elements of $G_{d}$ acting directly on the paths or by the demands of nonself-inverse symmetry operators in $G_{t}$ (compare restriction 3 in the introduction). We can easily cite examples of the second kind of cause without the first being applicable (e.g., $G_{t}=4 / m ; G_{d}=\overline{2}$; $\left.[u v 0] \rightarrow 4^{-1}[u v 0]=[u v 0] \rightarrow \overline{4}[u v 0]\right)$.

The two paths related by nonself-inverse operators (eq. (1)) need not be related by any element of $G_{d}$. Conversely, however, we can prove that two paths related by a self-inverse element of $G_{d}$ are necessarily also related by a nonself-inverse operator as in eq. (1). Let $S_{d 1}$ be a self-inverse operator of $G_{d}$ which leaves $[u v w]$ invariant; the path $[u v w] \rightarrow S_{t}[u v w]$ must be equivalent to the path $[u v w] \rightarrow S_{d 1} S_{t}[u v w]$. If $S_{t}[u v w]$ is also on $S_{d 1}$ then two paths connect the same directions and so are considered together as a single path as previously discussed and no equivalence of different paths results. If $S_{t}[u v w]$ is not on $S_{d}$ then we can show that either $S_{t}$ is nonself-inverse or $S_{d 1} S_{t}$ is nonself-inverse. To prove this statement the four possibilities of self-inverse operators $1,2, \overline{1}, \overline{2}$ for $S_{d 1}$ must be considered in turn. The possibilities 1 and $\bar{l}$ can be dismissed at once because the former has no effect and the latter cannot be an element of any $\mathrm{G}_{d}$. For $S_{d 1}=2$ any mirror plane whose normal is $\|$ to $S_{d 1}$ will leave [uvw] invariant; a center, mirror plane, or other 2 axis $\perp S_{d 1}$ will generate $[\bar{u} \bar{v} \bar{w}]$ still on $S_{d 1}$. Thus the only selfinverse operators $S_{t}$ which will move $S_{t}[u v w]$ off $S_{d 1}$ are 2 or $\overline{2}$ axes inclined to $S_{d 1}$ whose product with $S_{d 1}$ is a nonself-inverse operator. An exactly analogous argument applies for $S_{d 2}=\overline{2}$. Note also that if any $\left|S_{d}\right|>2$ for noncubic $G_{t}$ all elements of $\langle u v w\rangle$ are coincident with $S_{d}$. No $S_{d} \in \mathrm{G}_{d}$ can then repeat any path $[u v w] \rightarrow S_{t}[u v w]$ other than into its inverse (i.e., the same path with the direction of motion reversed). We are, however, only concerned with the number of paths emanating from [uvw]. We conclude that any pair of paths related by a selfinverse operator is also related by a pair of nonselfinverse operators as in equation $l$ and so turn our attention to examining the latter.

If $\mathrm{G}_{t}$ contains no $\left|S_{t}\right|>2$, i.e., when $q=0$, no distinct paths can be equal, so $J=\frac{\mathrm{N}_{t}}{\mathrm{~N}_{d}}-1$. The total number of nonself-inverse operator pairs may not be effective in relating distinct paths when the $\langle u v w\rangle$ is a special direction set. For a general direction set $\langle u v w\rangle: S_{t 1}[u v w] \not \equiv S_{t 2}[u v w]$. However, for a special direction $\langle u v w\rangle$ it could be that $S_{t 1}[u v w] \equiv S_{t 2}[u v w]$. Two cases arise: (1) $S_{t 1}=S_{t 2}^{-1}$ and $S_{t 2}^{2}[u v w]=1$. This will apply if and only if [uvw] $\| S_{t}\left(\left|S_{t}\right|>2\right)$. For noncubic $\mathrm{G}_{t}$ all $S_{t}\left(\left|S_{t}\right|>2\right)$ must be parallel to each other, and for $\langle u v w\rangle \| S_{t}\left(\left|S_{t}\right|>2\right)$ the number $q_{e}$ of independently effective pairs of nonself-inverse operators is always zero. (2) The other special case is that in which $S_{t 1}$ and $S_{t 2}$ are not a mutually inverse pair, yet generate the same path emanating from [uvw]. Equation 3 , however, expresses all $\mathrm{N}_{d}$ possible ways of expressing a given path. It follows that the condition for this special case is:

$$
S_{t 1}=S_{t 2} S_{d}
$$


However, for $S_{t 1} \| S_{t 2}$, all 2 and $\overline{2} \perp S_{t}$ relate all $S_{t}$ to $S_{t}^{-1}$ only. For $\overline{2} \| S_{t}$, i.e., a mirror plane perpendicular to $S_{t}$, will relate:

$$
\begin{aligned}
4 & \rightleftarrows \overline{4}^{-1}, \\
3 & \rightleftarrows \overline{6}^{-1}, \\
\text { and } 6 & \rightleftarrows \overline{3}^{-1} .
\end{aligned}
$$

For $\langle u v w\rangle \perp S_{t}$ therefore $q=q_{e}$ if $G_{d}=2$ and $\frac{q}{2}=q_{e}$ if $G_{d}=m$ or $m m 2$.

In summary, therefore,

$$
J=\frac{N_{t}}{N_{d}}-q_{e}-1
$$

where $q_{e} \leqslant q$.

For noncubic $G_{t}$ and $\left|S_{t}\right|>2$.

If $S_{t} \|\langle u v w\rangle$

If $S_{t} \perp\langle u v w\rangle$ and $S_{t} \| \overline{2}$

$$
\begin{array}{r}
q_{e}=0 \\
-\quad q_{e}=2
\end{array}
$$$$
\text { If } S_{t} \perp\langle u v w\rangle \text { and } S_{t} \text { not } \| \overline{2} \quad q_{e}=q
$$$$
\text { If } S_{t} \wedge\langle u v w\rangle \neq n \pi / 2 \quad q_{e}=q \text {. }
$$

The value of $q_{e}$ can be expressed in words as the following count taken from the $q$ nonself-inverse operator pairs: A pair is counted if neither of its elements multiplied by any element of the defect symmetry equals either its own inverse or an element of a pair previously counted.

\section{Jump Rates for Special Defect Directions in Noncubic Point Groups of the Trap Site}

For special defect directions $\langle u v w\rangle$ in noncubic trap point groups $G_{t}$ an exhaustive list of the total number $J$, of distinct paths, is given in tables 2 and 3 relating to centrosymmetric and other $G_{t}$, respectively. The maximum and minimum number of jump rates needed for relaxation is also given in these tables. If the concepts involved are not adequately clarified in the introduction of this paper, the reader is referred to our previous publication [1]. It is noteworthy that for special positions in noncentrosymmetric point groups, $J_{\min }>2$ occurs only for $G_{t}=\overline{6} m 2$ and $G_{d}=m_{[0001]}$.

The last two columns of table 2 show additional figures in parentheses corresponding to relaxation jump rates needed after the population has been upset by an influence that itself was centrosymmetric such as homogeneous mechanical strain. For general positions these numbers are always one less than the corre-

\begin{tabular}{|c|c|c|c|c|c|c|c|c|}
\hline \multirow[b]{2}{*}{$G_{t}$} & \multirow[b]{2}{*}{$N_{t}$} & \multirow[b]{2}{*}{$G_{d}$} & \multirow[b]{2}{*}{$N_{d}$} & \multirow[b]{2}{*}{$\frac{N_{t}}{N_{d}}$} & \multirow[b]{2}{*}{$q_{e}$} & \multirow[b]{2}{*}{$J$} & $J_{\mathrm{Min}}$ & $J_{\text {Max }}$ \\
\hline & & & & & & & \multicolumn{2}{|c|}{$\begin{array}{l}\text { Figures in parentheses refer } \\
\text { to limitations for centro- } \\
\text { symmetric influence }\end{array}$} \\
\hline$\overline{1}$ & 2 & 1 & 1 & 2 & 0 & 1 & $\mathrm{l}(0)$ & $1(0)$ \\
\hline $2 / m$ & 4 & $\begin{array}{l}2 \\
m\end{array}$ & $\begin{array}{l}2 \\
2\end{array}$ & $\begin{array}{l}2 \\
2\end{array}$ & $\begin{array}{l}0 \\
0\end{array}$ & $\begin{array}{l}1 \\
1\end{array}$ & $\begin{array}{l}1(0) \\
1(0)\end{array}$ & $\begin{array}{l}1(0) \\
1(0)\end{array}$ \\
\hline$m m m$ & 8 & $\begin{array}{c}m \\
m m 2\end{array}$ & $\begin{array}{l}2 \\
4\end{array}$ & $\begin{array}{l}4 \\
2\end{array}$ & $\begin{array}{l}0 \\
0\end{array}$ & $\begin{array}{l}3 \\
1\end{array}$ & $\begin{array}{l}2(1) \\
1(0)\end{array}$ & $\begin{array}{l}2(1) \\
1(0)\end{array}$ \\
\hline$\overline{3}$ & 6 & 3 & 3 & 2 & 0 & 1 & $1(0)$ & $1(0)$ \\
\hline$\overline{3} m$ & 12 & $\begin{array}{c}m \\
2 \\
3 m\end{array}$ & $\begin{array}{l}2 \\
2 \\
6\end{array}$ & $\begin{array}{l}6 \\
6 \\
2\end{array}$ & $\begin{array}{l}2 \\
2 \\
0\end{array}$ & $\begin{array}{l}3 \\
3 \\
1\end{array}$ & $\begin{array}{l}1(1) \\
1(1) \\
1(0)\end{array}$ & $\begin{array}{l}2(1) \\
2(1) \\
1(0)\end{array}$ \\
\hline $4 / m$ & 8 & $\begin{array}{l}m \\
4\end{array}$ & $\begin{array}{l}2 \\
4\end{array}$ & $\begin{array}{l}4 \\
2\end{array}$ & $\begin{array}{l}1 \\
0\end{array}$ & $\begin{array}{l}2 \\
1\end{array}$ & $\begin{array}{l}1(1) \\
1(0)\end{array}$ & $\begin{array}{l}2(1) \\
1(0)\end{array}$ \\
\hline $4 / \mathrm{mmm}$ & 16 & $\begin{array}{c}m_{[100]} \text { or } m_{[110]} \\
m_{[001]} \\
m m 2 \\
4 m m\end{array}$ & $\begin{array}{l}2 \\
2 \\
4 \\
8\end{array}$ & $\begin{array}{l}8 \\
8 \\
4 \\
2\end{array}$ & $\begin{array}{l}2 \\
1 \\
1 \\
0\end{array}$ & $\begin{array}{l}5 \\
6 \\
2 \\
1\end{array}$ & $\begin{array}{l}2(1) \\
2(2) \\
1(1) \\
1(0)\end{array}$ & $\begin{array}{l}3(2) \\
3(2) \\
2(1) \\
1(0)\end{array}$ \\
\hline $6 / m$ & 12 & $\begin{array}{l}m \\
6\end{array}$ & $\begin{array}{l}2 \\
6\end{array}$ & $\begin{array}{l}6 \\
2\end{array}$ & $\begin{array}{l}2 \\
0\end{array}$ & $\begin{array}{l}3 \\
1\end{array}$ & $\begin{array}{l}1(1) \\
1(0)\end{array}$ & $\begin{array}{l}2(1) \\
1(0)\end{array}$ \\
\hline $6 / \mathrm{mmm}$ & 24 & $\begin{array}{c}m_{[10 \overline{1} 0]} \text { or } m_{[2 \bar{\pi} 10]} \\
m_{[0001]} \\
m m 2 \\
6 m m\end{array}$ & $\begin{array}{r}2 \\
2 \\
4 \\
12\end{array}$ & $\begin{array}{r}12 \\
12 \\
6 \\
2\end{array}$ & $\begin{array}{l}4 \\
2 \\
2 \\
0\end{array}$ & $\begin{array}{l}7 \\
9 \\
3 \\
1\end{array}$ & $\begin{array}{l}2(1) \\
2(2) \\
1(1) \\
1(0)\end{array}$ & $\begin{array}{l}3(2) \\
3(2) \\
2(1) \\
1(0)\end{array}$ \\
\hline
\end{tabular}
sponding one for noncentrosymmetric influences as previously discussed [1], except for the minimum number of paths in point groups having a $\overline{3}$ axis, for which the numbers in parentheses did not differ from

TABLE 2. Paths for centrosymmetric noncubic trap symmetries; all special positions of point defects 
TABLE 3. Paths for noncentrosymmetric noncubic trap symmetries; all special positions of point defects

\begin{tabular}{|c|c|c|c|c|c|c|c|c|}
\hline$G_{t}$ & $N_{t}$ & $G_{d}$ & $N_{d}$ & $\frac{N_{t}}{N_{d}}$ & $q_{e}$ & $J$ & $J_{\text {Min }}$ & $J_{\text {Max }}$ \\
\hline 1 & 1 & 1 & 1 & 1 & 0 & 0 & 0 & 0 \\
\hline 2 & 2 & 2 & 2 & 1 & 0 & 0 & 0 & 0 \\
\hline$m$ & 2 & $m$ & 2 & 1 & 0 & 0 & 0 & 0 \\
\hline 222 & 4 & 2 & 2 & 2 & 0 & 1 & 1 & 1 \\
\hline$m m 2$ & 4 & $\begin{array}{c}m \\
m m 2\end{array}$ & $\begin{array}{l}2 \\
4\end{array}$ & $\begin{array}{l}2 \\
1\end{array}$ & $\begin{array}{l}0 \\
0\end{array}$ & $\begin{array}{l}1 \\
0\end{array}$ & $\begin{array}{l}1 \\
0\end{array}$ & $\begin{array}{l}1 \\
0\end{array}$ \\
\hline 3 & 3 & 3 & 3 & 1 & 0 & 0 & 0 & 0 \\
\hline 32 & 6 & $\begin{array}{l}2 \\
3\end{array}$ & $\begin{array}{l}2 \\
3\end{array}$ & $\begin{array}{l}3 \\
2\end{array}$ & $\begin{array}{l}1 \\
0\end{array}$ & $\begin{array}{l}1 \\
1\end{array}$ & $\begin{array}{l}1 \\
1\end{array}$ & $\begin{array}{l}1 \\
1\end{array}$ \\
\hline $3 m$ & 6 & $\begin{array}{c}m \\
3 m\end{array}$ & $\begin{array}{l}2 \\
6\end{array}$ & $\begin{array}{l}3 \\
1\end{array}$ & $\begin{array}{l}1 \\
0\end{array}$ & $\begin{array}{l}1 \\
0\end{array}$ & $\begin{array}{l}1 \\
0\end{array}$ & $\begin{array}{l}1 \\
0\end{array}$ \\
\hline 4 & 4 & 4 & 4 & 1 & 0 & 0 & 0 & 0 \\
\hline$\overline{4}$ & 4 & 2 & 2 & 2 & 0 & 1 & 1 & 1 \\
\hline 422 & 8 & $\begin{array}{l}2 \\
4\end{array}$ & $\begin{array}{l}2 \\
4\end{array}$ & $\begin{array}{l}4 \\
2\end{array}$ & $\begin{array}{l}1 \\
0\end{array}$ & $\begin{array}{l}2 \\
1\end{array}$ & $\begin{array}{l}1 \\
1\end{array}$ & $\begin{array}{l}2 \\
1\end{array}$ \\
\hline $4 \mathrm{~mm}$ & 8 & $\begin{array}{c}m \\
4 m\end{array}$ & $\begin{array}{l}2 \\
8\end{array}$ & $\begin{array}{l}4 \\
1\end{array}$ & $\begin{array}{l}1 \\
0\end{array}$ & $\begin{array}{l}2 \\
0\end{array}$ & $\begin{array}{l}1 \\
0\end{array}$ & $\begin{array}{l}2 \\
0\end{array}$ \\
\hline$\overline{4} 2 m$ & 8 & $\begin{array}{c}2 \\
m \\
m m 2\end{array}$ & $\begin{array}{l}2 \\
2 \\
4\end{array}$ & $\begin{array}{l}4 \\
4 \\
2\end{array}$ & $\begin{array}{l}1 \\
1 \\
0\end{array}$ & $\begin{array}{l}2 \\
2 \\
1\end{array}$ & $\begin{array}{l}1 \\
1 \\
1\end{array}$ & $\begin{array}{l}2 \\
2 \\
1\end{array}$ \\
\hline 6 & 6 & 6 & 6 & 1 & 0 & 0 & 0 & 0 \\
\hline$\overline{6}$ & 6 & $\begin{array}{l}m \\
3\end{array}$ & $\begin{array}{l}2 \\
3\end{array}$ & $\begin{array}{l}3 \\
2\end{array}$ & $\begin{array}{l}1 \\
0\end{array}$ & $\begin{array}{l}1 \\
1\end{array}$ & $\begin{array}{l}1 \\
1\end{array}$ & $\begin{array}{l}1 \\
1\end{array}$ \\
\hline 622 & 12 & $\begin{array}{l}2 \\
6\end{array}$ & $\begin{array}{l}2 \\
6\end{array}$ & $\begin{array}{l}6 \\
2\end{array}$ & $\begin{array}{l}2 \\
0\end{array}$ & $\begin{array}{l}3 \\
1\end{array}$ & $\begin{array}{l}1 \\
1\end{array}$ & $\begin{array}{l}2 \\
1\end{array}$ \\
\hline $6 \mathrm{~mm}$ & 12 & $\begin{array}{c}m \\
6 m\end{array}$ & $\begin{array}{r}2 \\
12\end{array}$ & $\begin{array}{l}6 \\
1\end{array}$ & $\begin{array}{l}2 \\
0\end{array}$ & $\begin{array}{l}3 \\
0\end{array}$ & $\begin{array}{l}1 \\
0\end{array}$ & $\begin{array}{l}2 \\
0\end{array}$ \\
\hline$\overline{6} m 2$ & 12 & $\begin{array}{c}m_{[10 \overline{10}]} \\
m_{[0001]} \\
m m 2 \\
3 m\end{array}$ & $\begin{array}{l}2 \\
2 \\
4 \\
6\end{array}$ & $\begin{array}{l}6 \\
6 \\
3 \\
2\end{array}$ & $\begin{array}{l}1 \\
1 \\
1 \\
0\end{array}$ & $\begin{array}{l}3 \\
4 \\
1 \\
1\end{array}$ & $\begin{array}{l}1 \\
2 \\
1 \\
1\end{array}$ & $\begin{array}{l}2 \\
2 \\
1 \\
1\end{array}$ \\
\hline
\end{tabular}

that corresponding to noncentrosymmetric influences. For special positions, however, there are additional causes for equalities of numbers in and out of parenthesis while in point groups containing a $\overline{3}$ axis a difference in minimum numbers of paths is obtained when the special direction is parallel to that $\overline{3}$-axis.

\section{References}

[1] J. B. Wachtman, Jr., and H. S. Peiser, Symmetry conditions on jump rates occurring in relaxation times associated with point defect motion between equivalent general sites in crystals, J. Phys. Chem. Solids 27, 975 (1966).

[2] A. S. Nowick, Dielectric and anelastic relaxation of crystals containing point defects, II. Adv. in Phys. (Phil. Mag. Suppl.) 16, 1 (1967).

[3] H. S. Peiser and J. B. Wachtman, Jr., Reduction of crystallographic point groups to subgroups by homogeneous stress, J. Res. NBS 69A (Phys. and Chem.) No. 4, 309 (1965).

[4] N. F. M. Henry and K. Lonsdale, Eds., International Tables for X-ray Crystallography (Kynoch Press, Birmingham, England, 1952). 